\title{
A COMPLETE SET OF POSTULATES FOR THE THEORY OF
}

\section{ABSOLUTE CONTINUOUS MAGNITUDE*}

\author{
$\mathrm{BY}$ \\ EDWARD V. HUNTINGTON
}

The following paper presents a complete set of postulates or primitive propositions from which the mathematical theory of absolute continuous magnitude can be deduced.

The fundamental concept involved is that of a rule of combination according to which any two objects of a given assemblage, in a definite order, determine a third object which itself belongs to the assemblage. (The same idea is the fundamental concept in the definition of a group.)

The rule of combination here considered is one upon which six fundamental restrictions are imposed, these requirements beging expressed in the postulates $\dagger$ 1 to 6.

The object of the work which follows is to show that these six postulates form a complete set; that is, they are (I) consistent, (II) sufficient, (III) independent (or irreducible). By these three terms we mean: (I) there is at least one assemblage in which the chosen rule of combination satisfies all the six requirements; (II) there is essentially only one such assemblage possible; (III) none of the six postulates is a consequence of the other five.

This being shown (theorems I, II, III), the postulates 1-6 may be said to define a single assemblage, which we shall call the system of absolute continuous magnitude. The rule of combination may then be called addition.

From another point of view, the propositions 1-6 may be accepted as expressing in precise mathematical form the essential characteristics of magnitude in the popular sense of the word; from this point of view they may properly be called the axioms $\uparrow$ of (absolute continuous) magnitude.

From either point of view, the propositions 1-6 form a complete logical basis for a deductive mathematical theory.

* Presented to the Society February 22, 1902. Received for publication March 5, 1902.

† Following the usual distinction, we use "postulate" to mean a proposition the acceptance of which is demanded or agreed upon as a basis for future reasoning, reserving "axiom" to mean "a self-evident proposition, requiring no formal demonstration to prove its truth, but received and accepted as soon as mentioned." 
The most recent work on this subject is a long and critical paper by $O$. HöLDER (see bibliographical note *) to which the present article is closely related.

* Bibliographical Note. - The following references include papers in which the properties of magnitude are deduced from any system of postulates, whether or not the independence of the postulates is discussed.

G. Veronese, Il continuo rettilineo e l'assioma V. d'Archimede, A tti della R. A ccad. de i Lincei, Memoired.cl.d. scienze fis., mat. e nat., ser. 4, vol. 6 (1889), pp. 603-624.

R. Betrazzi, Teoria delle grundezze, $4^{\circ}(1890)$, vii +181 pp., Pisa. Awarded a prize by the R. Accad. dei Lincei. Reviewed in Darboux's Bulletin dessciences mathématiques, ser. 2 , vol. 15 (1891), pp. 53-68.

O. Stolz, Ueber das Axiom des Archimedes, Mathematische Annalen, vol. 39 (1891), pp. 107-112.

C. Burali-Forti, Sulla teoria delle grandezze, Formulaire de Mathématiques, vol. 1 (1895), pp. 28-57. Written in Peano's mathematical language. See also Rivista di Matematica, vol. 3 (1893), pp. 76-101.

G. Veronese, Sul postulato della continuitd, Atti della R. Accad. dei Lincei, Rendiconti d. cl. d. Scienze fis, mat. e nat., ser. 5, vol. $6_{2}$ (1897), pp 161-168.

L. Couturat, Sur la définition du continu, Revue de metaphysique et de morale, vol. 8 (1900), pp. 157-168.

D. Hilbert, Ueber den Zuhlbegriff, Jahresbericht der deutschen MathematikerVereinigung, vol. 8 (1900), pp. 180-184.

E. V. Huntington, Die Grund-Operationen an absoluten und complexen Grössen, Braunschweig (1901), $63 \mathrm{pp}$.

O. Stolz und J. A. Gueiner, Theoretische Arithmetik, Leipzig (1901). A second edition, now in publication, of STolz's Allgemeine Arithmetik, 1885, 1886.

O. Hölner, Die Axiome der Quantität und die Lehre vom Mass, Lei pziger Berich te, Math.Phys. Classe, vol. 53 (1901), pp. 1-64.

HöLDER's main set of postulates, like the sets used in all the earlier papers, admits the idea of "greater and less" as a fundamental concept. In a footnote on p. 5, however, he works out, partially, a second set of postulates which does not involve this idea. The set of postulates adopted in the present paper is nearly the same as this second set of HöLDER's, with the exception that DEDEKIND's postulate of continuity is here replaced by WEIERSTRASS's, and that two of HöLDER's postulates, viz., [5] and the secoud part of [2], are here found to be deducible from the others. I am indebted to HöLDER's paper for many valuable suggestions, especially for the proof of the commutative law (see 22, Case II, p. 271), which in all the earlier papers is admitted as a fundamental law.

The three following works, which I have not seen, may also contain matter bearing on this subject :

C. Burali-Forti, Les propriétés formelles des opérations algébriques, Turin (1900). See also Rivista di Matematica, vol. 6 (1899) pp. 141-177.

R. Betrazzi, Grandezza, quantità e numero, Il Bolletino di Matematiche e di Scienze fisiche e naturali, Bologna, vol. 1 (1900), pp. 7-10, 70-73.

F. Aморео, Arithmetica particolure e generale, Naples (1900).

A complete set of postulates for the theory of positive integers with zero, in which the fundamental concept is that of an element successire to a given element, is given by

G. Peano, Sul concetto di numero, Rivista di Matematica, vol. 1 (1891), pp. 87-102, 256-267. See also Formulaire de Mathématiques, vol. 3 (1901), pp. 39-44. Essentially these postulates are reproduced in the footnote on p. 266.

A complete set of postulates for the theory of all whole numoers (positive, negative and zero) is given in two papers by

A. PADOA: 1) Essai d'une théorie algébrique des nombres entiers, presented to the International Congress of Philosophy, Paris, 1900, and reviewed in Rivista di Matematica, vol. 7 


\section{Fundamental concepts.}

A class of objects is determined when any condition is given such that every object in the universe must either satisfy or not satisfy the condition. Every object which satisfies the condition is said to belong to the class.

(The condition might be such that no object would belong to the class; this case, however, we shall agree to exclude from the discussion-that is, we shall consider those classes only which contain at least one object.)

A class thus defined is usually called, in mathematical parlance, an assemblage (Menge, ensemble), every object which belongs to the class being called an element of the assemblage.*

A rule of combination in an assemblage is any rule or agreement by which, when any two elements (whether the same or different) are given, in a definite order, some object (which may or may not itself belong to the assemblage $\dagger$ ) is uniquely determined.

If the first of the two given elements is denoted by $a$ and the second by $b$, then the object which they determine is denoted by $a \circ b$-read: " $a$ with $b$."

The notation $x=y$ indicates that the two symbols $x$ and $y$ are used to represent the same object, while $x \neq y$ indicates that the objects represented by $x$ and $y$ are different.

In the proofs of theorems I and III illustrations are drawn from the ordinary artificial systems of real and complex numbers. In the rest of the paper, however, only the natural system of ordinal numbers $\ddagger$ is presupposed.

(1901), pp. 73-84; 2) Un nouveau système irréductible des postulats pour l'algèbre, presented to the International Congress of Mathematicians, Paris, 1900, reviewed in the Bulletin of the American Mathematical Sooiety, 2d ser., vol. 7 (1900), p. 70. I have seen only the reviews of these papers. The fundamental concepts involved are the idea of an element successive to a given element, and the idea of an element symmetrical to a given element.

* H. Weber, Algehra, vol. 2 (1899), p. 4.

† Compare postulate 1 .

$\ddagger$ The system of ordinal numbers is an assemblage characterized by the following fundamental properties, which Peano (loc. cit.) has shown to be independent of one another :

$\left.1^{\circ}\right)$ The assemblage coutains a peculiar element, called the "first" ordinal number, and denoted by 1.

$2^{\circ}$ ) Every element, $m$, of the assemblage determines uniquely an object $m^{+}$, which itself belongs to the assemblage. This number $m+$ is called the number next following the number $\mathrm{m}$. (The number $1+$ is denoted by $2 ; 2^{+}$by 3 , etc.)

$3^{\circ}$ ) Whatever the number $m$ may be, the number next following $m$ is never 1 .

$4^{\circ}$ ) If the numbers next following two numbers $m$ and $n$ are the same, then $m$ and $n$ are the same.

$5^{\circ}$ ) If $S$ is any assemblage such that: $(a)$ the number 1 belongs to $S$, and $(b)$ the number next following $m$ belongs to $S$ whenever the number $m$ belongs to $S$; then $S$ contains every ordinal number.

From these fundamental propositions follows the validity of the common method of "mathematical induction." (See also 12.) 
If any law is given by which every ordinal number $k(k=1,2,3, \ldots)$ determines a definite object $\left(a_{k}\right)$ the objects so determined are said to form an "infinite sequence," in notation $a_{1}, a_{2}, a_{3}, \ldots, a_{k}, a_{k+1}, \ldots$ It should be noticed, however, that $a_{p}$ and $a_{q}(p \neq q)$ do not necessarily represent different objects, since, according to the given law, it may be that an object is repeated many times in the sequence.

In the present paper we are concerned with the assemblages in which the rule of combination satisfies certain fundamental conditions, expressed in the six postulates of the following paragraph.

The postulates of magnitude.

1. Whatever elements $a$ and $b$ may be ( $a=b$ or $a \neq b), a \circ b$ is also an element of the assemblage.

2. $a \circ b \neq a$.

3. $(a \circ b) \circ c=a \circ(b \circ c)$, whenever $a \circ b, b \circ c,(a \circ b) \circ c$ and $a \circ(b \circ c)$ belong to the assemblage.

4. When $a \neq b$ at least one of the following conditions is satisfied: either $\left.1^{\circ}\right)$ there is an element $x$ such that $a=b \circ x$, or $2^{\circ}$ ) there is an element $y$ such that $a$ o $y=b$.

5.* (Let the notation $a<b$ indicate that an element $y$ exists such that $a \circ y=b$; and let $a \leqq b$ indicate : $a<b$ or $a=b$.)

If $S$ is any infinite sequence of elements $\left(a_{k}\right)$, such that

$$
a_{k}<a_{k+1}, \quad a_{k}<c \quad(k=1,2,3, \cdots)
$$

(where $c$ is some fixed element), then there is one and only one element $A$ having the following two properties:

$\left.1^{\circ}\right) a_{k} \leqq A$ whenever $a_{k}$ belongs to $S$;

$2^{\circ}$ ) if $y$ and $A^{\prime}$ are such that $y \circ A^{\prime}=A$, then there is at least one element of $S$, say $a_{r}$, for which $A^{\prime}<a_{r}$.

6. Whatever element a may be, there are two elements $x$ and $y$ such that $x \circ y=a$; that is, in the notation explained in 5 , there is an element $x$ such that $x<a$.

\section{Consistency of the postulates.}

Theonem I.-The postulates 1-6 are consistent with each other.

To establish this theorem we have simply to exhibit some assemblage in which all the postulates are satisfied. Such an assemblage is the system of positive

* This postulate 5 is essentially the same as the principle employed by WeIERSTRASs, in his lectures, for the definition of an irrational number. But instead of saying, under $2^{\circ}$ ), "if $A^{\prime}<A$," that is : "if a $y$ exists such that $A^{\prime} \circ y=A$," I have inverted the order of the $A^{\prime}$ and $y$. (Cf. 24.) 
real numbers, when $a \circ b$ is defined as $a+b$. Another such assemblage is the system of positive real numbers greater than 1 , when $a \circ b$ is defined as $a \times b$. (See also 36.)

Development of the theory in preparation for the proof of theorem II.

Remarks. We notice that the two systems mentioned in the proof of theorem I are equivalent (simply isomorphic); that is, the first can be put into one-to-one correspondence with the second in such a way that when $a$ corresponds with $a^{\prime}$ and $b$ with $b^{\prime}$ then $a \circ b$ will correspond with $a^{\prime} \circ b^{\prime}$. In theorem II we shall show that every assemblage which satisfies the postulates 1-6 will be equivalent with these. The propositions 7-35, which we proceed to deduce from the fundamental propositions 1-6, will prepare the way for the proof of theorem II.

\section{Greater and less. Fundamental inequalities.}

7. Definition. If with respect to two elements $a, b$ an element $x$ exists such that $a=b \circ x$, we shall write: $a>b(a$ greater than $b)$ and $b<a$ (b less than $a$ ).

8. Of the three relations $a=b, a>b, a<b$ of any two elements $a$ and $b$

$\left.1^{\circ}\right)$ not more than one can be true, and

$2^{\circ}$ ) at least one must be true.

The proof for the first part is indirect.

9. If $a>b$ and $b>c$ then $a>c . \dagger$

10. $a \circ b>a$.

11. If $b>b^{\prime}$ then $a \circ b>a \circ b^{\prime}$.

For, from $b=b^{\prime} \circ x$ it follows by 1 and 3 that $a \circ b=\left(a \circ b^{\prime}\right) \circ x$.

\section{Multiples.}

12. Ordinal numbers. On the basis of the fundamental properties mentioned in the introduction, we state here briefly such other properties of ordinal numbers as we shall have occasion to use. $\ddagger$

a) Definition. The sum, $p+q$, of two numbers, $p$ and $q$, is the number defined by the following recurrent formulæ:

$$
\begin{gathered}
p+1=p^{+} ; p+2=(p+1)+1 ; p+3=(p+2)+1 ; \cdots ; \\
p+(k+1)=(p+k)+1 .
\end{gathered}
$$

b) $p+q=q+p . \quad$ (Proof by mathematical induction.)

* With every theorem will be indicated the fundamental propositions from which it is deduced.

† From 8 and 9 we see that any assemblage which satisfies 1, 2, 3, 4 will possess Grössen rharakter in the sense defined by Schönflies, Jahresbericht derdeutschen MathematikerVereinigung, vol. 8 (1900), Bericht über die Mengenlehre, p. 15.

$\ddagger$ The most accessible proofs are in Stolz and GMeiner, loc. cit., pp. 12-23. 
c) Definition. The product, $q p$, of two numbers, $p$ and $q$, is the number defined by the following recurrent formulæ:

$$
1 p=p ; 2 p=1 p+p ; 3 p=2 p+p ; \cdots ;(k+1) p=k p+p .
$$

d) $q p=p q . \quad$ (Proof by mathematical induction.)

e) If $p \neq q$, then there is either a number $\lambda$ such that $p=q+\lambda$ or else a number $\mu$ such that $q=p+\mu$ (and not both). In the first case $p$ is said to come later, in the second case earlier, than $q$ in the sequence of ordinal numbers.

13. Multiples.

a) Definition. The mth multiple, ma, of any element $a$ ( $m$ being any ordinal number) is the element defined by the following recurrent formulæ:

$$
1 a=a ; 2 a=1 a \circ a ; \quad 3 a=2 a \circ a ; \ldots ; \quad(k+1) a=k a \circ a .
$$

All the multiples of any element $a$ clearly belong to the assemblage, by 1 ; but it should be noticed that there is nothing in postulate 1 to prevent some or all of these multiples from being the same. (See 13f.)

b) $p a \circ q a=(p+q) a$.

The proof by induction with respect to $q$ involves $13 a, 3,12 a$.

c) $p a \circ q a=q a \circ p a$. (By 12b and 13b.)

d) $q(p a)=(q p) a$.

The proof by induction as to $q$ involves $13 a, 12 c, 13 b$.

e) $q(p a)=p(q a)=q p a=p q a . \quad(\mathrm{By} .12 d$ and 13d.)

f) $p a=q a$ when and only when $p=q$.

For, if $p=q$, then $p a=q a$, by identity. Conversely, if $p a=q a$, then $p=q$; for, suppose $p \neq q$, then by $12 e$ either $p=q+\lambda$ or $q=p+\mu$, whence, by hypothesis and $13 b$, either $q a=q a \circ \lambda a$ or $p a=p a \circ \mu a$, relations each impossible, by 2 .

g) $p a>q a$ when and only when $p$ comes later than $q$. In particular, $p a \geqq a$.

For, if $p$ comes later than $q$, then by $12 e$ and $13 b, p a=q a \circ \lambda a$, whence, by 7, $p a>q a$. Conversely, if $p a>q a$, then $p$ comes later than $q$; for suppose $p$ earlier than $q$, or else $p=q$, then by $12 e, 13 b, 13 f$ we should have either $q a=p a \circ \mu a$ or else $p a=q a$, relations each impossible by $8,1^{\circ}$ ).

$$
\text { h) }(b \circ p a) \circ(q a \circ c)=(b \circ q a) \circ(p a \circ c) . \quad(\text { By 13c and 3.) }
$$

Principle of Archimedes. Further inequalities.

14. Principle of Archimedes. Whatever elements $a$ and $b$ may be, there is a multiple of $a$, sıy $m a$, such that $m a>b$.

$(1,2,3,4,5)$ 
The proof is indirect, as follows: Suppose the theorem to be false; then, by $8, n a \leqq b$, or, by 10 and $9, n a<2 b$, for every multiple of $a$. Now by $13 g$ the successive multiples of $a$ form an infinite sequence of elements each of which is less than the succeeding one. From this fact and the supposition $n a<2 b$ follows by 5 the existence of an element $A$ having the following two properties:

$\left.1^{\circ}\right) n a \leqq A$ for every multiple of $a$;

$2^{\circ}$ ) if $y . \circ A^{\prime}=A$ there is at least one multiple of $a$, say $n^{\prime} a$, such that $n^{\prime} a>A^{\prime}$.

From $1^{\circ}$ ) it follows that $a<A$, by $13 g$ and 9 ; therefore there is by 7 an element $A^{\prime}$ such that $a \circ A^{\prime}=A$. Hence, by $2^{\circ}$ ), we have $n^{\prime} a>A^{\prime}$ for at least one multiple of $a$. From this relation follows, by 11, $a \circ n^{\prime} a>a \circ A^{\prime}$, that is, $a \circ n^{\prime} a>A$, whence, by $13 c$ and $13 a,\left(n^{\prime}+1\right) a>A$. This last result, however, is in contradiction with $\left.1^{\circ}\right)$. Therefore the theorem must be true.

15. If $a<b$ there are two successive multiples of $a$, say pa and $(p+1) a$, such that

$$
p a<b \leqq(p \circ 1) a . \quad(1,2,3,4,5)
$$

For, there is one multiple of $a$, viz., $1 a$, which is, by hypothesis, less than $b$. Hence, if it were true that $(p+1) a<b$ whenever $p a<b$, it would follow by mathematical induction that $m a<b$ for every multiple of $a$, a result impossible by 14 .

16. $a \circ b>b$.

$(1,2,3,4,5)$

When $a \geqq b$ the theorem is clearly true, by 10 and 9 .

When $a<b$ we have, by 15, $p a<b \leqq(p+1) a$. From $p a<b$ follows, by $11, a \circ p a<a \circ b$, or, by $13 c$ and $13 a(p+1) a<a \circ b$. But $b \leqq(p+1) a$; hence, by $9, b<a \circ b$.

17. If $a>a^{\prime}$ then $a \circ b>a^{\prime} \circ b$. (By 7, 1, 3, 16, 11.) $\quad(1,2,3,4,5)$

18. If $a>a^{\prime}$ and $b>b^{\prime}$ then $a \circ b>a^{\prime} \circ b^{\prime}$. $\quad(1,2,3,4,5)$

(By 11, 17, 9.)

19. Let $a \circ b=a^{\prime} \circ b^{\prime}$; then from $b=b^{\prime}$ follows $a=a^{\prime}$, and from $b>b^{\prime}$ follows $a<a^{\prime}$.

$(1,2,3,4,5)$

For the other suppositions (8) lead to contradictions with 17, 11 or 18.

20. If $a>a^{\prime}$ then $m a>m a^{\prime}$.

The proof by induction involves 18 and $13 a$.

21. If $m a=m a^{\prime}$ then $a=a^{\prime}$; if $m a>m a^{\prime}$ then $a>a^{\prime} . \quad(1,2,3,4,5)$

For the other suppositions (8) lead to contradictions with 20.

The commutative law.

22. Commutative law.

$$
a \circ b=b \circ a \text {. }
$$


It is clear that in any assemblage which satisfies 1-5, the postulate 6 must be either true or false. By considering both cases, we shall prove the commutative law from 1, 2, 3, 4, 5 without using 6 .

Case $I$. Let postulate 6 be false; then the given assemblage contains a minimum element $E$, such that whatever element $a$ may be $a \geqq E$. Then every element of the assemblage is some multiple of $E$.

For, suppose there were an element $c$ not a multiple of $E$; then, since $E<c$, we have, by $15, p E<c<(p+1) E$. Hence, by $7, p E \circ y=c$ and $c \circ z=(p+1) E$, whence $(p E \circ y) \circ z=(p+1) E$, or, by 3 and $13 a$, $p E \circ(y \circ z)=p E \circ E$. But from this follows, by $19, y \circ z=E$, or, by 7 , $y<E$, which is impossible in this case.

Since every element is thus of the form $m E$, the commutative law follows at once from $13 c$.

Case II.* Let postulate 6 be true ; then whatever element $a$ may be, there are wo elements $x$ and $y$ such that $x \circ y=a$. The proof for this case is indirect.

Suppose $a \circ b<b \circ a$; then, by 7, $(a \circ b) \circ y=b \circ a$.

By 6 take $y^{\prime} \circ y^{\prime \prime}=y$, and $x$ less than the least of the four elements $y^{\prime}, y^{\prime \prime}$, $a, b$. From $x<y^{\prime}$ and $x<y^{\prime \prime}$ follows, by $18, x \circ x<y^{\prime} \circ y^{\prime \prime}$, that is,

$$
x \circ x<y \text {. }
$$

Since $x<a$ and $x<b$, we can take $p$ and $q$ by 15 such that

and

$$
p x<a \leqq(p+1) x
$$

$$
q x<b \leqq(q+1) x .
$$

From the first parts of these inequalities, we have, by 18, $p x \circ q x<a \circ b$, whence, by 17, $(p x \circ q x) \circ y<(a \circ b) \circ y$, that is :

$$
(p x \circ q x) \circ y<b \circ a \text {. }
$$

From the second parts of the same inequalities, reversing the order, we have, by 18 and $13 a, b \circ a \leqq(q x \circ x) \circ(p x \circ x)$, or by $13 h$

whence by 11 and 9

$$
b \circ a \leqq(q x \circ p x) \circ(x \circ x) \text {, }
$$

$$
b \circ a<(q x \circ p x) \circ y \text {. }
$$

These two results, A) and B), are, however, inconsistent, by 8; for by $13 c$

$$
p x \circ q x=q x \circ p x
$$

therefore the supposition from which they were deduced is false.

In a similar way we can show that the supposition $a \circ b>b \circ a$ is also false. Hence by 8 the third possibility, viz., $a \circ b=b \circ a$, must be true.

* Cf. Holder, loc. cit., p. 13.

Trans. Am. Math. Soc. 18 
23. $(b \circ x) \circ(y \circ c)=(b \circ y) \circ(x \circ c)$.

$(1,2,3,4,5)$

The proof is the same as the proof of $13 h$, when 22 is used in place of $13 c$.

\section{Limit of an increasing sequence.}

24. If $S$ is any infinite sequence of elements $\left(a_{k}\right)$, each of which is less than the succeeding one, and also less than some fixed element $c$ :

$$
a_{k}<a_{k+1}, \quad a_{k}<c \quad(k=1,2,3, \cdots),
$$

then there is one and only one element $A$ having the following two properties:

$\left.1^{\circ}\right) a_{k} \leqq A$ whenever $a_{k}$ belongs to $S$;

$\left.2^{\circ}\right)$ if $A^{\prime}<A$ there is at least one element of $S$, say $a_{r}$, for which $A^{\prime}<a_{r}$.

The element $A$ thus determined is called the limit of the infinite sequence $S$ and is denoted by $\lim \left(a_{k}\right)$.

$(1,2,3,4,5)$

Proof. Whenever $A^{\prime}<A$ there is an element $y$ such that $y \circ A^{\prime}=A$; for by 7 there is an element $y$ such that $A^{\prime} \circ y=A$, and by 22

$$
A^{\prime} \circ y=y \circ A^{\prime} \text {. }
$$

Therefore by 5 there is at least one element $A$ which has the properties $1^{\circ}$ ) and $2^{\circ}$ ). Further two elements $A, B$ each with both these properties are the same. For suppose $B<A$. Then by $2^{\circ}$ ) as to $A$ there is an element of $S$ which is greater than $B$, while by $1^{\circ}$ ) as to $B$ no element of $S$ is greater than $B$.

25. If $\lim \left(a_{k}\right)=A$ and $\lim \left(b_{k}\right)=B$ then $\lim \left(a_{k} \circ b_{k}\right)=A \circ B$.

We consider here two cases, as under 22.

$$
(1,2,3,4,5)
$$

Case $I$. Let postulate 6 be false. Then there is a minimum element $E$ such that, whatever element $a$ may be, $a \geqq E$; whence, as we saw in 22 , Case I, every element is some multiple of $E$. In this case, by $13 g$, there cannot be an infinite number of elements less than a fixed element $c=m E$, and hence the conditions in the hypothesis cannot be fulfilled.

Case II. Let postulate 6 be true. Then whatever element $a$ may be, there are two elements $x$ and $y$ such that $x \circ y=a$. The first condition $1^{\circ}$ ) for the truth of the theorem is in this case clearly satisfied; for by hypothesis and $\left.24,1^{\circ}\right) a_{k} \leqq A$ and $b_{k} \leqq B$; hence, by $18, a_{k} \circ b_{k} \leqq A \circ B$ for every index $k$.

To show that the second condition, $2^{\circ}$ ), is also satisfied, let $C^{\prime}$ be any element $<A \circ B$. By 7, $C^{\prime} \circ y=A \circ B$; by 6 take $y^{\prime} \circ y^{\prime \prime}=y$ and $x$ less than the least of the elements $y^{\prime}, y^{\prime \prime}, A, B$; and by 7 and 22 take $A^{\prime}$ and $B^{\prime}$ such that $A^{\prime} \circ x=A, B^{\prime} \circ x=B$. Hence $\left(A^{\prime} \circ x\right) \circ\left(B^{\prime} \circ x\right)=A \circ B$, that is, by $23,\left(A^{\prime} \circ B^{\prime}\right) \circ(x \circ x)=C^{\prime} \circ\left(y^{\prime} \circ y^{\prime \prime}\right)$, while, by $18, x \circ x<y^{\prime} \circ y^{\prime \prime}$. Therefore by $19, A^{\prime} \circ B^{\prime}>C^{\prime}$.

But by $7, A^{\prime}<A$ and $B^{\prime \prime}<B$; therefore by $24,2^{\circ}$ ) there is an element 
$a_{s}>A^{\prime}$ and an element $b_{t}>B^{\prime}$. Let $r$ be an index that comes later than $s$ and $t$; then by $9, a_{r}>A^{\prime}$ and $b_{r}>B^{\prime}$, whence, by $18, a_{r} \circ b_{r}>A^{\prime} \circ B^{\prime}$.

Therefore, by $9, a_{r} \circ b_{r}>C^{\prime}$, and the theorem is established.

26. If $\lim \left(a_{k}\right)=A$ then $\lim \left(m a_{k}\right)=m A$.

$(1,2,3,4,5)$

The inductive proof involves $13 a$ and 25 .

\section{Submultiples.}

27. Lemma. Given a and $m$ there is an element $y$ such that $m y<a$. $(1,2,3,4,5,6)$

If the theorem is true when $m=n$, we shall have, by $7, n z \circ z^{\prime}=a$, and by 6 we can take $y$ less than $z$ and $z^{\prime}$. Then, by $20, n y<n z$ and, by 18, $n y \circ y<n z \circ z^{\prime}$, whence, by $13 a,(n+1) y<a$; that is, the theorem will be true when $m=n+1$. But it is clearly true when $m=1$, by 6 ; hence it will be true for every value of $m$.

28. Given any element $a$ and any ordinal number $m$ there is one and only one element $x$ such that $m x=a$.

The element $x$ thus determined is called the mth submultiple of $a$ and is denoted by $a / m$. Thus $m(a / m)=a$.

$(1,2,3,4,5,6)$

The proof in outline is this: we construct an infinite sequence of elements $x_{1}, x_{2}, x_{3}, \ldots$, which has by 24 a limit $x$; we then prove that the sequence $m x_{1}, m x_{2}, m x_{3}, \ldots$ has the limit $a$, whence it follows that $x$ is the element required in the theorem.

a) The first element of the sequence is found as follows:

Take $y_{1}$ by 27 so that $m y_{1}<a$, and then $p_{1}$ by 15 so that

$$
p_{1}\left(m y_{1}\right)<a \leqq\left(p_{1}+1\right)\left(m y_{1}\right) ;
$$

hence, by $13 e$ and $13 a, m\left(p_{1} y_{1}\right)<a \leqq m\left(p_{1} y_{1}\right) \circ m y_{1}$.

Then for the first element of the sequence we take $x_{1}=p_{1} y_{1}$, and by 7 we have $m x_{1} \circ e_{1}=a$.

$b)$ When any element, as the $k$ th, has been found, the next following element 一the $(k+1)$ th-is obtained as follows :

We have

By 27 take $y_{k+1}$ so that

whence, by $13 g$ and 16 ,

$$
m x_{k} \circ e_{k}=a .
$$

Then take $p_{k+1}$ by 15 so that $p_{k+1}\left(m y_{k+1}\right)<a \leqq\left(p_{k+1}+1\right)\left(m y_{k+1}\right)$, whence by $13 e$ and $13 a$

$$
m\left(p_{k+1} y_{k+1}\right)<a \leqq m\left(p_{k+1} y_{k+1}\right) \circ m y_{k+1} \text {. }
$$


For the $(k+1)$ th element we then take $x_{k+1}=p_{k+1} y_{k+1}$, and by 7 we have

$$
m x_{k+1} \circ e_{k+1}=a \text {. }
$$

c) In the sequence thus determined we have from (4) and (5)

hence by 8 and 11

$$
m\left(x_{k+1}\right) \circ e_{k+1} \leqq m\left(x_{k+1}\right) \circ m y_{k+1} ;
$$

$$
e_{k+1} \leqq m y_{k+1} \text {. }
$$

From (6) and (3) we have, by 9, $e_{k+1}<e_{k}$, while from (1) and (5

Hence by 19 and 10

(7) and by $21,13 g$ and 9

$$
\begin{gathered}
m x_{k+1} \circ e_{k+1}=m x_{k} \circ e_{k}=a . \\
m x_{k}<m x_{k+1}<a, \\
x_{k}<x_{k+1}<a .
\end{gathered}
$$

Further, if $c$ is any element, there is an index $r$ such that $e_{r}<c$. For, if $c \leqq e_{k}$ for every index $k$, then $c \leqq e_{k+1}$, or by $20,(6),(2)$, and (3)

$$
(k+1) c \leqq(k+1) e_{k+1} \leqq(k+1) m y_{k+1}<e_{k}<a
$$

for every ordinal number $k+1$, which is impossible by 14 .

From this it follows that if $a^{\prime}<a$ then there is an index $r$ such that

$$
a^{\prime}<m x_{r} \text {. }
$$

For, let $a^{\prime} \circ z=a$ by 7 and take $e_{r}<z$. Then by (1) $a^{\wedge} \circ z=m x_{r} \circ e_{r}$, whence, by $19, a^{\prime}<m x_{r}$.

d) The theorem is now readily established; for, from (8) it follows by 24 that the sequence $x_{1}, x_{2}, x_{3}, \ldots$ has a limit: $\lim \left(x_{k}\right)=x$; hence by 25 $\lim \left(m x_{k}\right)=m x$. But from (7) and (9) we have by $24 \lim \left(m x_{k}\right)=a$. Therefore $m x=a$. That no other element, $x^{\prime}$, can have this property follow at once from 21.

29. If $p$ comes later than $q$ in the sequence of ordinal numbers (12e), then $a / p<a / q$. In particular, $a / p \leqq a$.

$(1,2,3,4,5,6)$

For, suppose $a / p \geqq a / q$; then, by $13 g$ and $20, p(a / p)>q(a / p) \geqq q(a / q)$, whence, by $28, a>a$, which is impossible.

30. Whatever elements $a$ and $b$ may be, there is a submultiple of $a$, say $a / n$, such that $a_{i} m<b$. (By 8, 20, 28, 14.)

$(1,2,3,4,5,6)$

\section{Rational fractions.}

31. Definition. Any multiple (13a) of any submultiple (28) (or any submultiple of any multiple) of an element $a$, is called a rational fraction of $a$; in notation $p a / q$. $(1,2,3,4,5,6)$ 
This (ambiguous) notation is allowable, for by $13 e$ and 28 we have $q[p(a / q)]=p[q(a / q)]=p a$, whence, by 28 ,

$$
p(a / q)=(p a) / q \text {. }
$$

32. The following theorems are deduced from 31 by the aid of $12,13,21$ and 28.

$(1,2,3,4,5,6)$

a) $m p a / m q=p a / q$.

For, $m q(m p a / m q)=m p a=m q(p a / q)$, by $13 e$ and 28 . Hence follows the theorem, by 21.

b) $p a / q \circ p^{\prime} a / q^{\prime}=\left(q^{\prime} p+q p^{\prime}\right) a / q q^{\prime}$.

For, $p a / q=q^{\prime} p\left(a / q q^{\prime}\right)$ and $p^{\prime} a / q^{\prime}=q p^{\prime}\left(a / q q^{\prime}\right)$, by $\left.a\right)$ and 12d. Hence follows the theorem, by $13 b$.

c) $p a / q=p^{\prime} a / q^{\prime}$ when and only when $q^{\prime} p=q p^{\prime}$.

d) $p a i q>p^{\prime} a / q^{\prime}$ when and only when $q^{\prime} p$ comes later than $q p^{\prime}$.

These two theorems follow by $13 f$ and $13 g$ from the equations given under $b$ ).

e) If $p a / q<p^{\prime} a / q^{\prime}$ there is a rational fraction of $a$, say $m a / n$, such that $p a / q \circ m a / n=p^{\prime} a / q^{\prime}$.

Since by $d) q^{\prime} p$ comes earlier than $q p^{\prime}$, there is by $12 e$ an ordinal number $\mu$ such that $q^{\prime} p+\mu=q p^{\prime}$, and if we take $m a / n=\mu a / q q^{\prime}$, we shall satisfy the theorem. For by $a), 12 d$ and $13 b$

$p a / q \circ m a / n=q^{\prime} p\left(a / q q^{\prime}\right) \circ \mu\left(a / q q^{\prime}\right)=\left(q^{\prime} p+\mu\right) a / q q^{\prime}=q p^{\prime} a / q q^{\prime}=p^{\prime} a / q^{\prime}$.

33. Given $c$, and $a<b$, we can find $m c / n$ such that $a<m c / n<b$.

$$
(1,2,3,4,5,6)
$$

Let $a \circ y=b$, by 7 . By 30 and 16 take $c / n<y<b$, and by 15 take $m$ so that $m(c / n)<b \leqq(m+1)(c / n)$. Then $a<m c / n$. For, if $a \geqq m c / n$, then, by $18, a \circ y>m c / n \circ c / n$, or, by $13 a, b>(m+1) c / n$, which is impossible.

34. Given $m c / n<a \circ b$, we can find $p c / q<a$ and $p^{\prime} c / q^{\prime}<b$ such that $p c / q \circ p^{\prime} c / q^{\prime}=m c / n$.

$(1,2,3,4,5,6)$

To fix our ideas, let $a \leqq b$.

If $m c / n \leqq b$, take $c / q<a$ and $<m c / n$, by 30 , and by $32 e$ take $p^{\prime} c / q^{\prime}$ so that $c / q \circ p^{\prime} c / q^{\prime}=m c / n$. Then by 16 and $9, p^{\prime} c / q^{\prime}<b$.

If $b<m c / n$, we have, by 7 and $22, y \circ b=m c / n<a \circ b$, whence, by 17, $y<a$. By 33 and 9 take $p c / q$ so that $y<p c / q<a \leqq b<m c / n$ and by $32 e$ take $p^{\prime} c / q^{\prime}$ so that $p c / q \circ p^{\prime} c / q^{\prime}=m c / n=y \circ b$, whence, by $19, p^{\prime} c / q^{\prime}<b$.

Upper limit of an assemblage of rational fractions.

35. If $U$ is any assemblage of rational fractions of a such that every element of $U$ is less than some fixed element $c$, then there is one and only one element $A$ having the following two properties:

$1^{\circ}$ ) every element of $U$ is $\leqq A$;

$\left.2^{\circ}\right)$ if $a^{\prime}<A$, there is at least one element of $U$ which is $>a^{\prime}$. 
The element $A$ thus deternined is called the upper limit of the assemblage $U$, and is denoted by $\overline{\mathbf{L}}(U)$.

Proof:

$(1,2,3,4,5,6)$

Case I.-Suppose the assemblage $U$ contains a maximum element; then this maximum element will clearly be the element $A$ required in the theorem.

Case II.-Suppose on the other hand that $U$ does not contain a maximum element. Then when any element of $U$ is given we can find another element of $U$ greater than the given one; whence it follows that $U$ is an infinite assemblage.

To establish the theorem in this case we make use of a device due to G. CAN TOR by which the assemblage of all the rational fractions of $a$ can be arranged in an infinite sequence, $R$, as follows :

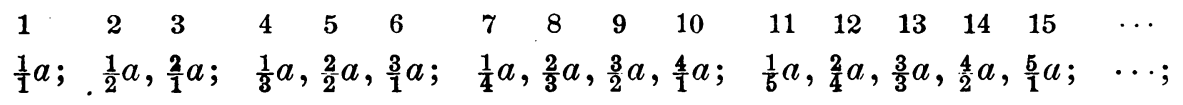

Taking this complete sequence $R$ we first cast out every element which does not belong to $U$; what is left is a sequence $U^{\prime}$ containing all the elements of $U$ and no others. $U^{\prime}$ will be an infinite sequence, since $U$ is an infinite assemblage, but its elements will not, in general, be arranged in order of magnitude.

Out of the sequence $U^{\prime}$ we then select the elements $x_{1}, x_{2}, x_{3}, \ldots$ as follows : $x_{1}$ is the first element of $U^{\prime} ; x_{2}$ is the first element beyond $x_{1}$ which is greater than $x_{1} ; x_{3}$ is the first element beyond $x_{2}$ which is greater than $x_{2}$; and so on; in general, $x_{k+1}$ is the first element of $U^{\prime}$ which is $>x_{k}$. (When any element $x_{k}$ of this sequence is given, the next element, $x_{k+1}$, can surely be found, since otherwise $x_{k}$ would be a maximum element, which contradicts the hypothesis.)

The elements $x_{1}, x_{2}, x_{3}, \ldots$ thus determined form an infinite sequence, $s$, in which every element is less than the succeding one, while all (being elements of $U$ ) are less than the fixed element $c$. Hence by 24 the sequence $S$ determines a definite element $A$ as its limit: $\lim \left(x_{k}\right)=A$.

Now first, every element of $U$ will be $\leqq A$. For, suppose $p a / q>A$ were an element of $U$, and hence occupied a place in the sequence $U^{\prime}$. Then either $p a / q$ would itself belong to $S$, or else some earlier element of $U^{\prime}$, which is $\geqq p a / q$, would belong to $S$; in either case we should have an element of $S$ greater than $A$, which is impossible, by $24,1^{\circ}$ ).

And secondly, if $A^{\prime}<A$, there is an element of $U$ which is $>A^{\prime}$. For, by $24,2^{\circ}$ ), there is an element of $S$ which is $>A^{\prime}$, and this element belongs also to $U$.

Therefore the element $A$ satisfies both the conditions in the theorem.

Further, no other element can satisfy both these conditions. (Proof as in the latter part of 24.) Hence the theorem is true in all cases. 
Sufficiency of the postulates to define an assemblage.

Theorem II.-Any two assemblages $M$ and $M^{\prime}$ which satisfy the postulates 1-6 are equivalent; that is, they can be brought into one-to-one correspondence in such a way that $a \circ b$ will correspond with $a^{\prime} \circ b^{\prime}$ whenever $a$ and $b$ in $M$ correspond with $a^{\prime}$ and $b^{\prime}$ in $M^{\prime}$ respectively.

First, take any element $E$ of $M$ and any element $E^{\prime}$ of $M^{\prime}$, to serve as "unit elements."

a) To every element a of $M$ we can assign a definite element $a^{\prime}$ of $M^{\prime}$, as follows:

Let $U$ be the assemblage of all rational fractions of $E$ which are less than $a,{ }^{*}$ and let $U^{\prime}$ be the assemblage comprising the same rational fractions of $E^{\prime}$. (That is, $p E^{\prime} / q$ is to belong to $U^{\prime}$ when, and only when, $p E / q$ belongs to $U$.) Now by 9 every element of $U$ is less than some fixed rational fraction $m E / n$ (where $a<m E / n$ ). Therefore by $32 d$ every element of $U^{\prime}$ will be less than $m E^{\prime} / n$. Hence by 35 the assemblage $U^{\prime}$ will have an upper limit, $a^{\prime}$; this element $a^{\prime}$ we then assign to $a$. We have then $\overline{\mathbf{L}}(U)=a$ and $\overline{\mathbf{L}}\left(U^{\prime}\right)=a^{\prime}$.

b) Similarly to every element of $M^{\prime}$ we assign a definite element of $M$.

c) Further, if $a^{\prime}$ is the element assigned to a, then a will be the element assigned to $a^{\prime}$.

To prove this it is sufficient to show that every rational fraction of $E^{\prime}$ which is less than $a^{\prime}$ belongs to $U^{\prime}$. Thus, let $p E^{\prime} / q$ be any such fraction. Then by $\left.24,2^{\circ}\right)$, since $\overline{\mathrm{I}}\left(U^{\prime}\right)=a^{\prime}$, there is an element of $U^{\prime}$, say $\lambda E^{\prime} / \mu$, for which $p E^{\prime} / q<\lambda E^{\prime} / \mu$, whence, by $32 d, p E / q<\lambda E / \mu$. But $\lambda E ; \mu<a$, since otherwise $\lambda E^{\prime} / \mu$ could not belong to $U^{\prime}$; hence, by $9, p E / q<a$, and therefore $p E^{\prime} / q$ belongs to $U^{\prime}$. Thus a strictly one-to-one correspondence is established between $M$ and $M^{\prime}$.

d) To prove the second part of the theorem, let $U, V$ and $W$ be the assemblages comprising all the rational fractions $E$ which are less than $a, b$ and $a \circ b$ respectively.

Then, if $x$ belongs to $U$ and $y$ to $V, x \circ y$ will belong to $W$, by $32 b$ and 18. And if $z$ belongs to $W$ we can find an $x$ of $U$ and a $y$ of $V$ such that $x \circ y=z$, by 34 .

Thus the assemblage $W$ is composed wholly of elements of the form $x \circ y$.

Similarly, if $U^{\prime}, V^{\prime} W^{\prime}$ are the assemblages comprising all rational fractions of $E^{\prime}$ which are less than $a^{\prime}, b^{\prime}, a^{\prime} \circ b^{\prime}$ respectively, then $W^{\prime}$ will be composed wholly of elements of the form $x^{\prime} \circ y^{\prime}$, where $x^{\prime}$ and $y^{\prime}$ belong respectively to $U^{\prime}$ and $V^{\prime}$.

Now by hypothesis $U$ and $U^{\prime}$ (and also $V$ and $V^{\prime}$ ) comprise the same rational fractions of $E$ and $E^{\prime}$ respectively. Hence, by $32 b, W$ and $W^{\prime}$ will also comprise the same rational fractions of $E$ and $E^{\prime}$ respectively; that is, $a^{\prime} \circ b^{\prime}$ will correspond with $a \circ b$.

* Cf. Dedekind's idea of a cut (Schnitt) in his Stetigkeit und irrationale Zahlen. 
36. From Theorems I and II we may say that the postulates 1-6 define essentially a single assemblage. This assemblage we call the system of absolute continuous magnitude, and the rule of combination addition.

For example, the two systems of artificial numbers mentioned in the proof of Theorem I are systems of magnitude in the sense here defined.

Another example (if we admit the usual geometric axioms) is the system of distances laid off along a line in one direction from a fixed point, $a \circ b$ being defined as the combined length of $a$ and $b$, or, equally well, as the length of the hypotenuse of the right triangle whose legs are $a$ and $b . *$

\section{Independence of the postulates.}

Theorem III.-The postulates 1-6 are independent; that is, no one of them is a consequence of the other five.

The independence of any one of the postulates will be proved if we can find any assemblage which satisfies all the other postulates, but not the one in question. $\dagger$ We give below an assemblage of the required sort for each of the six postulates, thus establishing the theorem.

$M_{1}$ ) Let $M_{1}$ be the assemblage of all positive real numbers less than 10 : $0<x<10$; and let $a \circ b$ be defined as $a+b$. Then $M_{1}$ satisfies all the other postulates, but not 1 .

Postulate 1 is clearly not satisfied, since, for example, $5 \circ 8=13$ is not an element of the assemblage. The other postulates all hold.

$M_{2}$ ) Let $M_{2}$ be the assemblage of all real numbers, while $a \circ b=a+b$. Then $M_{2}$ satisfies all the other postulates, but not 2 .

Postulate 2 does not hold, for $a \circ 0=a$. Postulates 1, 3, 4 and 6 clearly hold. In 5 notice that whatever elements $a$ and $b$ may be there is always an element $y$ such that $a \circ y=b$, so that by the definition of the symbol $<$, we shall have $a<b$ for all values of $a$ and $b$. Hence $S$ may be any infinite sequence of elements, and any element will answer for $c$; then 5 is satisfied, since any element may be taken as $A$.

$M_{3}$ ) Let $M_{3}$ be the assemblage of all real numbers, while $a \circ b$ is defined as follows: when $a \neq b, a \circ b=(a+b) / 2$; when $a=b, a \circ a=a+1$.

Then $M_{3}$ satisfies all the other postulates, but not 3 .

The third postulate does not hold, since, for example, $(2 \circ 6) \circ 10=7$, while $2 \circ(6 \circ 10)=5$. Postulate 1 is clearly satisfied; 2 also holds, since $(a \circ b) / 2 \neq a$ when $a \neq b$. In 4 we can take $y=2 b-a$ and in 6 we can take $x=a-1=y$; hence 4 and 6 are satisfied. In 5 notice that $a<b$ when-

* This last illustration is used by H. WeBER, loc. cit., vol. 1, p. 9.

† This method was employed by Peano (loc. cit.) and by Hilbert in his Grundlagen der Geometrie, Leipzig, 1899. 
ever $a \neq b$, since whenever $a \neq b$ there is an element $y$, viz., $y=2 b-a$, for which $a \circ y=b$. Hence $S$ may be any infinite sequence in which each element is different from the one next following; and $c$ may be any element not belonging to $S$. We can then take as $A$ the element $c$, and 5 is satisfied.

$M_{4}$ ) Let $M_{4}$ be the assemblage of all the couples of the form $(a, \beta)$ where $a$ and $\beta$ are positive real numbers; and let the rule of combination be the following:

$$
\left(a_{1}, \beta_{1}\right) \circ\left(a_{2}, \beta_{2}\right) \doteq\left(a_{1}+a_{2}, \beta_{1}+\beta_{2}\right) .
$$

Then $M_{4}$ satisfies all the other postulates, but not 4 .

(The couples $(\alpha, \beta)$ may be represented as the points in the first quadrant of the complex plane, excluding the axes.) To show that 4 does not hold, consider, for example, $a=(2,5), b=(2,4)$. Postulates $1,2,3$ and 6 clearly hold. In 5 notice that when $\left(a_{1}, \beta_{1}\right)<\left(a_{2}, \beta_{2}\right)$ then $a_{1}<a_{2}$ and $\beta_{1}<\beta_{2}$, according to the definition of the symbol $<$. Hence, under the conditions in 5 , the $a$ 's of the couples which belong to $S$ have an upper limit $\xi$, and the $\beta$ 's an upper limit, $\eta$. If now we take $A=(\xi, \eta)$ we see that 5 is satisfied.

$M_{5}$ ) Let $M_{5}$ be the assemblage of positive rational numbers, while $a \circ b=a+b$. Then $M_{5}$ satisfies all the other postulates, but not 5 .

Postulate 5 is not satisfied; for, consider any sequence of increasing rational fractions used to define any irrational number, as $\sqrt{\overline{2}}$. All the other postulates clearly hold.

$\left.M_{6}\right)$ Let $M_{6}$ be the assemblage of positive integers, while $a \circ b=a+b$. Then $M_{6}$ satisfies all the other postulates, but not 6 .

Postulate 6 clearly does not hold, since $M_{6}$ contains the minimum element 1 . Postulate 5 is satisfied; for no infinite sequence $S$ which satisfies the conditions stated can occur. All the other postulates clearly hold.

\section{Two unsolved problems.}

1. Suppose that in $5,2^{\circ}$ ) the condition: " $y \circ A^{\prime}=A$ " be replaced by: " $A^{\prime} \circ y=A$." Can 14 and 22 be deduced from the postulates in this form, or must a new postulate like this: * "Given $a$ and $b$, there is an $x$ such that $a \circ b=b \circ x$ "- be added? In the latter case, how is the independence of the new postulate to be proved?

2. Suppose that in 5 the "sequence $S$ " be replaced by: "any assemblage of elements all of which are $<c$." Is postulate 4 then deducible or still independent?

HARVARD UNIVERSITY,

Cambridge, Mass.

* Cf. HöldER, loc. cit., p. 5, footnote, axiom [5]. 